\title{
Outcome of Arthrocentesis in Patients with Internal Derangement of Temperomandibular Joint with Disc Reduction and without Disc Reduction
}

\author{
Authors \\ Manoj $\mathbf{S}^{\mathbf{1}}$, Ashik $\mathbf{A}^{2}$, Kavitha Janardanan ${ }^{3}$, Ajith Kumar $\mathbf{K}^{4}$, Sobhana $\mathbf{C} \mathbf{R}^{\mathbf{5}}$ \\ ${ }^{1}$ Additional Professor, Department of OMFS, Govt. Dental College, Thiruvananthapuram, Kerala \\ Email: manojsree@gmail.com \\ ${ }^{2}$ Maxillofacial Surgeon, Department of Dental Surgery, Health service, Palakkad, Kerala \\ ${ }^{3}$ Assistant Professor, Department of Prosthodontics, Govt. Dental College, Thiruvananthapuram \\ ${ }^{4}$ Professor, Department of OMFS, Govt. Dental College, Alappuzha, Kerala \\ ${ }^{5}$ Principal and Professor, Govt. Dental College, Thrissur, Kerala
}

\begin{abstract}
Aim: To compare the outcome of arthrocentesis in patients with internal derangement of temporomandibular joint, with disc reduction and without disc reduction.

Materials and Methods: Twenty patients were included in this study. Dynamic ultrasonography was done to assess the status of the temporomandibular joint disc. Patients were divided into 2 groups, those with disc reduction and without disc reduction. Arthrocentesis was done under local anesthesia. The outcome variables were pain level, inter incisal distance, and lateral excursion of the TMJ. Post-operative ultrasonography was taken to check whether arthrocentesis can release the closed lock of the articular disc.
\end{abstract}

Results: The results showed a statistically significant difference in the outcome between the two groups and the patients without disc reduction responded to the procedure well. Post-operative dynamic ultrasonography revealed no change in the status of the disc in patients without disc reduction.

Conclusion: Arthrocentesis gives better results in patients with internal derangement of temporomandibular joint without disc reduction. Even though there is a significant reduction in the symptoms associated with temporomandibular joint, arthrocentesis failed to release the closed lock of the articular disc, and disc recapturing is not essential to obtain good function and for relief of pain in patients with closed lock

Keywords: Temporomandibular Joint Arthrocentesis, TMJ pain, Mouth Opening, Closed lock, Disc reduction.

\section{Introduction}

TMJ problems are common presentations in most Oral and Maxillofacial Surgery Departments. After Nitzan and co-authors eloquently described the technique of arthrocentesis in 1991, it rapidly became popular for treating a subgroup of these

patients as the simplest form of surgical therapy with the aim of washing out inflammatory mediators, releasing the articular disc, and disrupting adhesions between the surface of the disc and the joint fossa by hydraulic pressure 
of the lavage solution. So, if a patient fails to respond to conservative measures, surgery is not usually indicated.

Arthrocentesis is the method of flushing out the temporomandibular joint by placing needles to the upper joint compartment using local anesthesia orsedation. The effectiveness of joint lavage may be explained by the joint space expansion achieved with the introduction of fluid and by the washing out of inflammatory mediators and catabolites. According to Dorrit W. Nitzan ${ }^{1}$, arthrocentesis is highly efficient in resolving signs and symptoms associated with disorders caused by adhering forces or friction that is eliminated by lavage, such as intermittent clicking, anchored disc phenomenon, and open lock, and is also efficient for releasing limited mouth opening and pain in approximately $70 \%$ of patients who have TMJ osteoarthritis. The outcomes are sufficiently effective to prevent further surgical intervention. In this study we investigate the efficacy of Arthrocentesis in relieving pain as measured by visual analog scale for pain, and improvement of mouth opening as measured in millimeters in patients with temporomandibular joint internal derangement with disk reduction and without disk reduction. The status of the disc is assessed preoperatively using dynamic ultrasonographic examination. In patients without disc reduction, postoperative ultrasonograph is taken to assess whether there is disk reduction due to arthrocentesis. Outcome variables are assessed and compared between the two groups.

\section{Patients, Materials and Methods}

The study was conducted in the department of OMFS, Government Dental College, Thiruvananthapuram. The study was designed as a longitudinal observational study. The study population was patients with TMJ internal derangement (TMJ ID) who failed to respond to conservative management of 2 months. The patients with bony degeneration and recurrent TMJ dislocations were excluded. Conservative management for temporomandibular joint disorders included participating in physical therapy, avoiding traumatic behaviors such as clenching or gum chewing, changing the patient's diet, administering non steroidal antiinflammatory drugs or muscle relaxants, and wearing occlusal appliances. After two months the failed cases were taken up for arthrocentesis. Dynamic ultrasonography was done to assess the status of discs. Based on the ultrasonography findings, patients are divided into 2 groups

Group 1- Patients without temporomandibular joint disc reduction

Group 2- Patients with temporomandibular joint disc reduction

The following criteria are studied,

1. Pain level assessed with visual analogue scale pre-operative, immediatepostoperative, 1 week, 1 month, and 6 month interval

2. Mandibular range of motion and mouth opening in millimeters.

3. Post-operative disc reduction, in patients without disc reduction preoperatively, using dynamic ultrasonography at one week post-operative follow up.

\section{Procedure}

Arthrocentesis is performed under local anesthesia. Patient is seated in a semi reclined position. An auriculotemporal nerve block and local infiltration to the areas for joint penetration is given using $2 \%$ lignocaine hydrochloride. A cantho-tragal line is drawn from the midpoint of the tragus to the lateral can thus of the ipsi lateral eye. A point is made 10 millimeters forward from the midpoint of the tragus and 2 millimeters inferior to the line. This is the point of placement of first needle. This point is confirmed by palpation with the patient opening and closing the mouth. A second point corresponding to the height of the articular eminence is marked at 20 millimeters anterior to the midtragal point and10 millimeters below the tragal-canthal plane. This is the point of placement of the second portal for the outflow of irrigant. The superior joint space is entered with an 18 gauge needle directed in 


\section{JMSCR Vol||05||Issue||12||Page 31956-31964||December}

ananterior-medial-inferior direction at the first point. Aim for the zygomatic arch and gently walk the needle into the superior joint space. The position of the needle is confirmed by mandibular movements. The patient is asked to keep the mouth open slightly during the procedure. A mouth prop can be used for the same. Once the joint space is entered, it is insufflated with Ringer's lactate solution. Several milliliters of solution are injected passively into the joint space till there is rebound of the syringe pressure with jaw movement. With the needle in place, the syringe is removed. A second needle is inserted for the outflow portal at the point described earlier. A 10 milliliter syringe with Ringer's lactate solution is then connected to the first needle, and fluid is injected to the superior joint space. The second needle provides outflowfor the solution. The lavage is performed by using approximately 100 milliliters of Ringer's lactate (RL) solution. After completion of the lavage, the joint is manipulated by moving the patient's mouth through opening, protrusive, and excursive movements to free up the disc. According to literature a goal of 30 to 40 millimeters inter incisal opening should be achieved at the completion of arthrocentesis. All the patients in both the study groups achieved more than $30 \mathrm{~mm}$ of inter incisal opening post-operatively. Patients were monitored on the day of procedure, 1 week, 1 month \& 6 months after the procedure. Dynamic ultrasonography is taken at 1 week post-operative follow up to assess the status of the disc.

\section{Results}

A prospective study was conducted on 20 patients who reported to the Department of Oral and Maxillofacial Surgery, Government Dental College, Thiruvananthapuram. All patients included in the study were followed fora period of 6 months, with no dropouts. Dynamic ultrasonography was taken from the Department of Radiodiagnosis, Government Medical College, Thiruvananthapuram, performed by single observer, for all the patients to assess the status of disc. Depending on the ultrasonographic finding, patients were categorized into two groups.

Group 1- Patients without temporomandibular joint disc reduction

Group 2- Patients with temporomandibular joint disc reduction

Ten patients were included in each group

Age

In our study, the mean age of patients in group 1 is $38.6 \pm 11.3$ and that of group 2 is $36.6 \pm 9$. Difference in mean age between the two groups is found to be statistically not significant. In group 1, $70 \%$ of the patients were below 40 years of age where as in group2, $50 \%$ of the patients were below 40 years of age.

Table:1 Comparison of age based on group

\begin{tabular}{|c|c|c|c|c|}
\hline \multirow{2}{*}{ Age } & \multicolumn{2}{|c|}{ Group 1 } & \multicolumn{2}{c|}{ Group 2 } \\
\cline { 2 - 5 } & Number & Percent & Number & Percent \\
\hline$<40$ & 7 & 70.0 & 5 & 50.0 \\
\hline$>=40$ & 3 & 30.0 & 5 & 50.0 \\
\hline Mean \pm SD & \multicolumn{2}{|c|}{$38.6 \pm 11.3$} & \multicolumn{2}{c|}{$36.6 \pm 9$} \\
\hline
\end{tabular}

$\mathrm{t}=0.44, \mathrm{p}=0.666$

Fig 1. Comparison of age based on group

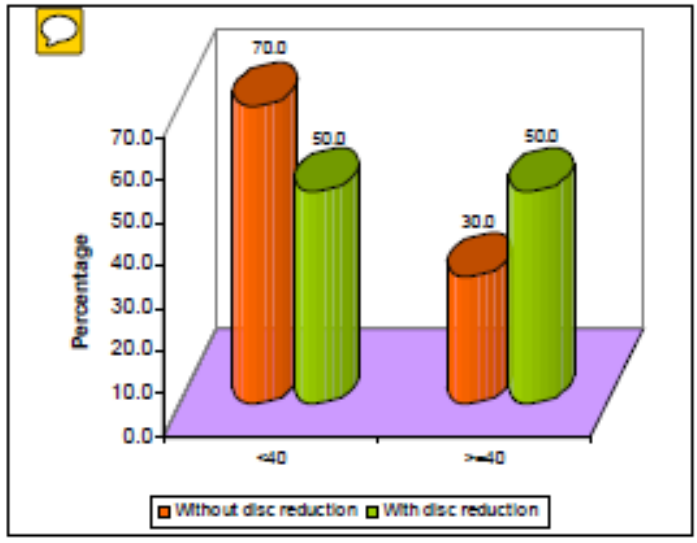

\section{Gender}

Temporomandibular joint internal derangements are more common in females. Current literature supports this gender difference. In our study, $90 \%$ of the patients in both group 1 and 2 were females. 
Table: 2 Comparison of gender based on group

\begin{tabular}{|c|c|c|c|c|c|}
\hline \multirow{2}{*}{ Gender } & \multicolumn{2}{|c|}{ Group 1 } & \multicolumn{2}{c|}{ Group 2 } & \multirow{2}{*}{$\mathrm{p} \#$} \\
\cline { 2 - 5 } & Number & Percent & Number & Percent & \\
\hline Male & 1 & 10.0 & 1 & 10.0 & \multirow{2}{*}{$\mathrm{p}>0.05$} \\
\hline Female & 9 & 90.0 & 9 & 90.0 & \\
\hline
\end{tabular}

\# : Fisher's Exact Test

Fig 2. Comparison of gender based on group

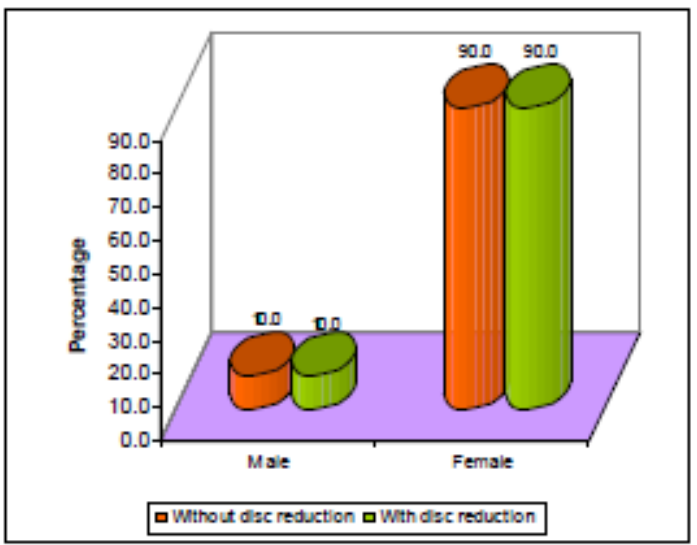

\section{Outcome measures}

1. Pain level assessed with visual analogue scale
2. Mouth opening- Inter incisal distance in millimeters

3. Lateral excursion in millimeters

4. Status of disc post operatively in group 1 patients (assessed by dynamicultrasonography, 1 week after arthrocentesis)

All the variables are measured (except ultrasonography) pre operatively, post operatively, immediately after 1 week, after 1 month and 6 months. Dynamic ultrasonography was taken pre operatively and 1 week post operatively

\section{Visual analogue scale for pain measurement}

Compared to group 2, there is a greater reduction of immediate post-operative pain, measured by VAS score, in group 1. Comparison of immediate post-operative pain, assessed by VAS score was statistically significant. In group 1 , there is a further reduction of pain after 1 week, 1 month and on $6^{\text {th }}$ month follow up. Three patients (1 male and 2 female) from group 1experienced total relief of pain post operatively. In group 2, there was not much reduction in the VAS score on postoperative follow up at 1 week, 1 month and 6 month follow Up.

Table: 3 Comparison of VAS pain level at different intervals between groups

\begin{tabular}{|l|c|c|c|c|c|c|c|c|}
\hline \multirow{2}{*}{$\begin{array}{l}\text { VAS pain } \\
\text { level }\end{array}$} & \multicolumn{3}{|c|}{ Group 1 } & \multicolumn{3}{c|}{ Group 2 } & \multirow{2}{*}{ t } & $\mathrm{p}$ \\
\cline { 2 - 8 } Pre-op & 7.6 & 0.5 & 10 & 7.6 & 0.5 & 10 & 0 & 1.000 \\
\hline $\begin{array}{l}\text { Imm post } \\
\text { op }\end{array}$ & 2.9 & 1.0 & 10 & 4.0 & 0.5 & 10 & $3.16^{* *}$ & 0.005 \\
\hline 1 week & 2.2 & 0.9 & 10 & 4.0 & 0.5 & 10 & $5.51^{* *}$ & 0.000 \\
\hline 1 month & 2.2 & 0.9 & 10 & 4.0 & 0.5 & 10 & $5.51^{* *}$ & 0.000 \\
\hline 6month & 2.2 & 0.9 & 10 & 4.0 & 0.5 & 10 & $5.51^{* *}$ & 0.000 \\
\hline
\end{tabular}

**:-Significant at 0.01 level 
Fig 3. Comparison of VAS pain level at different intervals between groups

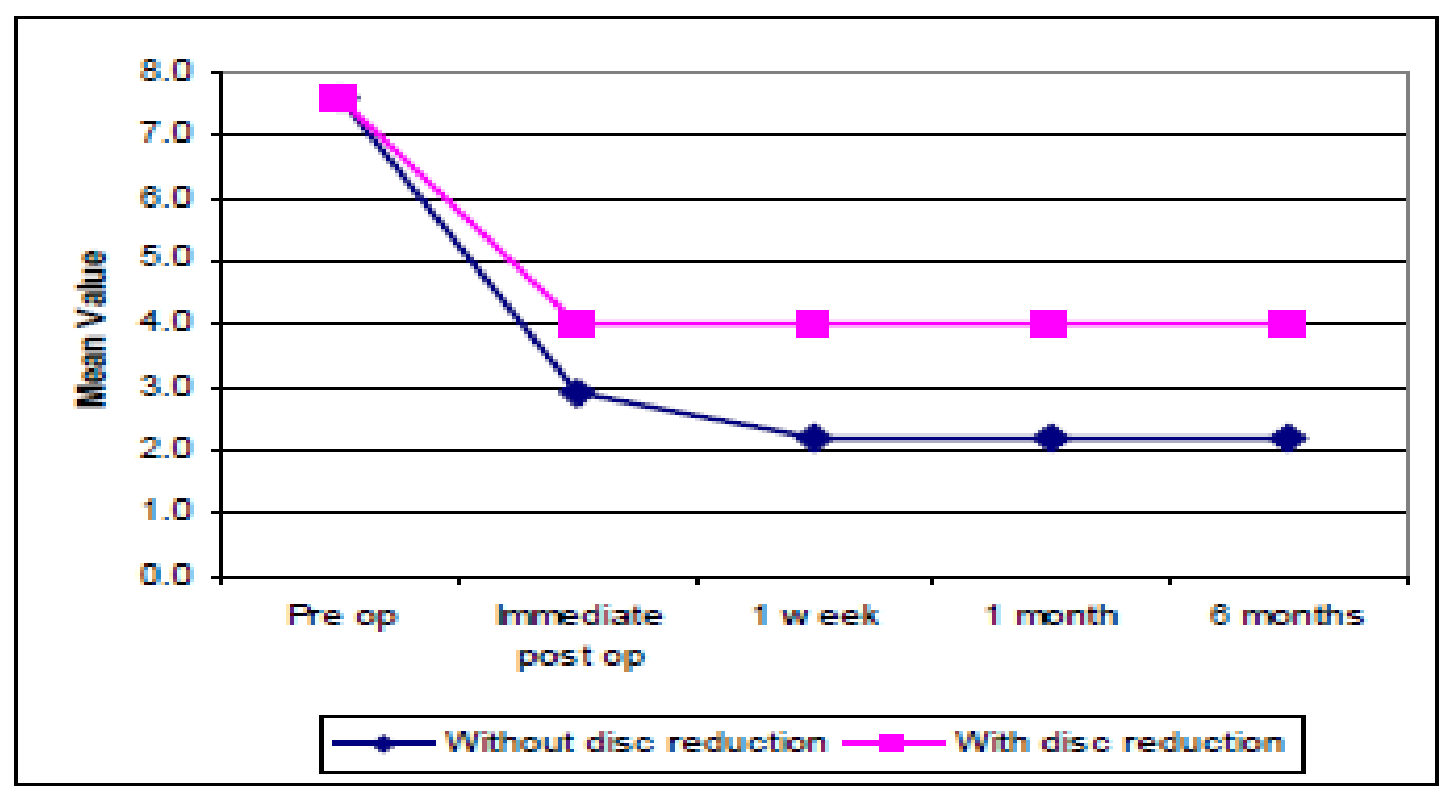

\section{Mouth opening}

Comparison of immediate post- operative inter incisal distance between the two groups was statistically insignificant. There was greater improvement in mouth opening in group 1compared to group 2, on follow up. In group 1 patients, there was more improvement in mouth opening during post- operative follow up period. Refer table 4 and figure 4.

\section{Lateral excursion}

Post-operative lateral excursion is more in group 1 than group 2 and the difference is statistically significant.
Post-operative dynamic ultrasonography of group 1 patients revealed no change in the statusof the disc following arthrocentesis. Even though there is a significant reduction in the symptoms associated with the temporomandibular joint, arthrocentesis failed to release the closed lock of the articular disc. According to Salvatore Sembronio et $\mathrm{al}^{2}$ disc recapturing is not essential to obtain good function and for relief of pain in patients with closed lock. Our results support this observation.

Table:4 Comparison of Inter incisal distance at different intervals between groups

\begin{tabular}{|l|c|c|c|c|c|c|c|c|}
\hline \multirow{2}{*}{$\begin{array}{l}\text { Inter incisal } \\
\text { distance }\end{array}$} & \multicolumn{3}{|c|}{ Group 1 } & \multicolumn{3}{c|}{ Group 2 } & \multirow{2}{*}{ t } & $\mathrm{p}$ \\
\cline { 2 - 8 } & Mean & SD & $\mathrm{N}$ & Mean & SD & $\mathrm{N}$ & 1.41 & 0.176 \\
\hline Pre-op & 22.7 & 3.0 & 10 & 20.6 & 3.7 & 10 & 1.7 \\
\hline $\begin{array}{l}\text { Immediate } \\
\text { post op }\end{array}$ & 37.0 & 3.3 & 10 & 34.3 & 2.5 & 10 & 2.08 & 0.052 \\
\hline 1week & 41.4 & 2.8 & 10 & 35.2 & 2.2 & 10 & $5.51^{* *}$ & 0.000 \\
\hline 1month & 41.6 & 3.2 & 10 & 35.0 & 2.1 & 10 & $5.44^{* *}$ & 0.000 \\
\hline 6month & 41.8 & 3.2 & 10 & 35.0 & 2.1 & 10 & $5.67^{* *}$ & 0.000 \\
\hline
\end{tabular}

**: - Significant at 0.01 level 
Fig 4.Comparison of Inter incisal distance at different intervals between groups

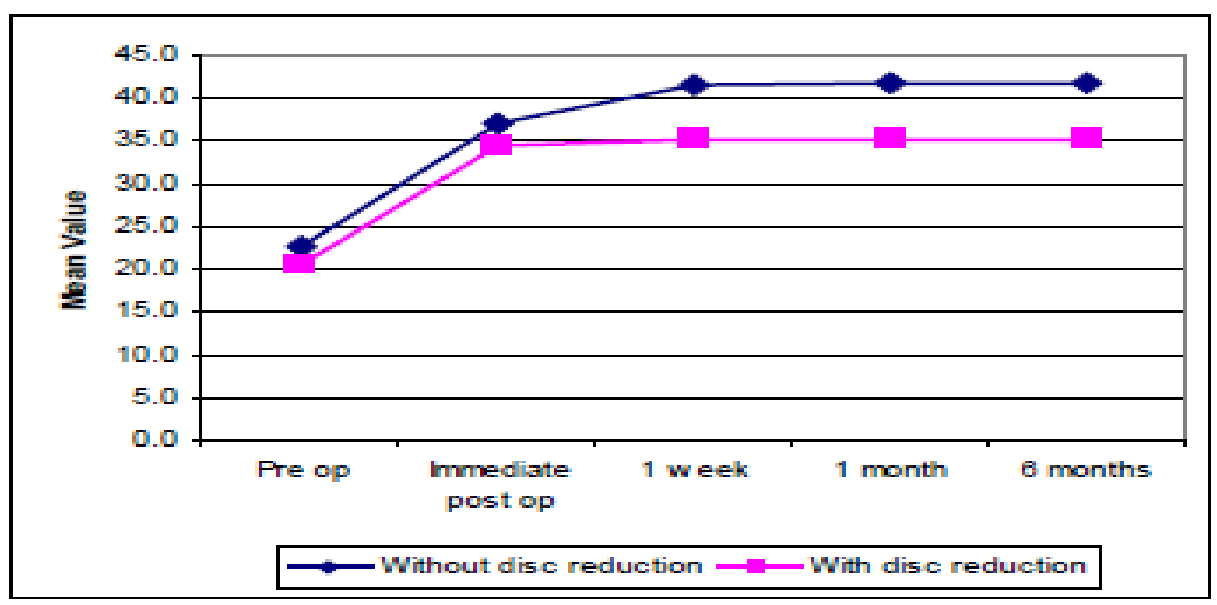

Table: 5 Comparison of Lateral excursion at different intervals between groups

\begin{tabular}{|c|c|c|c|c|c|c|c|c|}
\hline \multirow{2}{*}{$\begin{array}{l}\text { Lateral } \\
\text { excursion }\end{array}$} & \multicolumn{3}{|c|}{ Group 1} & \multicolumn{3}{|c|}{ Group 2} & \multirow[b]{2}{*}{$\mathrm{t}$} & \multirow[b]{2}{*}{$\mathrm{p}$} \\
\hline & Mean & SD & $\mathrm{N}$ & Mean & SD & $\mathrm{N}$ & & \\
\hline Pre-op & 2.3 & 0.5 & 10 & 2.5 & 0.5 & 10 & 0.88 & 0.388 \\
\hline $\begin{array}{l}\text { Immediate } \\
\text { post op }\end{array}$ & 5.5 & 0.5 & 10 & 4.8 & 0.4 & 10 & $3.28^{* *}$ & 0.004 \\
\hline 1 week & 5.6 & 0.5 & 10 & 4.8 & 0.4 & 10 & $3.79^{* *}$ & 0.001 \\
\hline 1 month & 5.6 & 0.5 & 10 & 4.8 & 0.4 & 10 & $3.79^{* *}$ & 0.001 \\
\hline 6month & 5.6 & 0.5 & 10 & 4.8 & 0.4 & 10 & $3.79^{* *}$ & 0.001 \\
\hline
\end{tabular}

**: - Significant at 0.01 level

Fig 5. Comparison of Lateral excursion at different intervals between groups

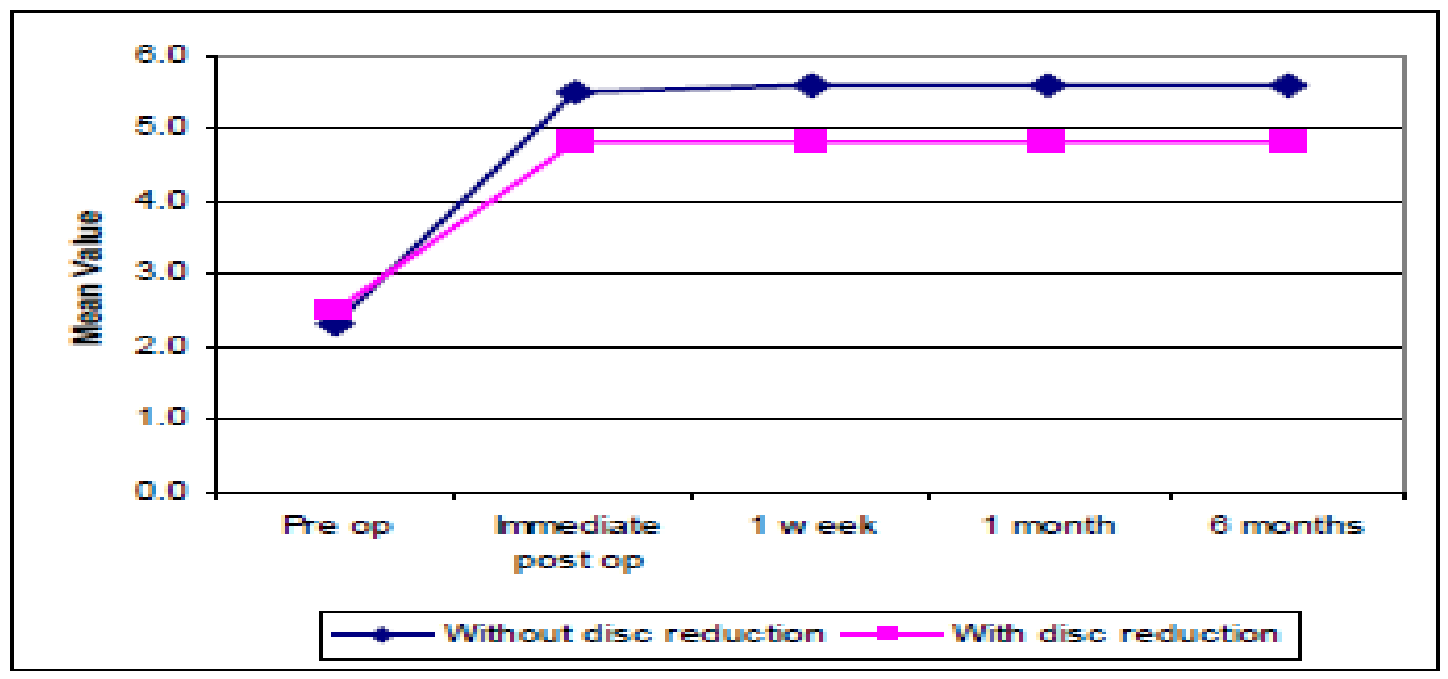

\section{Discussion}

Temporomandibular disorders are defined as clinical problems involving the masticatory musculature, the temporomandibular joints and associated structures, orboth. Articular disorders include disc displacement disorders, arthritic or degenerative changes, and neoplasm. Three cardinal signs of a temporomandibular disorder are (1) pain with mandibular function, (2) limitation of mandibular movement, and (3) joint sounds. 
Symptomatic temporomandibular joint disease is often accompanied by accumulation of inflammatory mediators within the synovial fluid. The accumulation of these inflammatory mediators may persist for some time within the joint space because of the relatively avascular nature of this space. The mechanism of arthrocentesis to lavage the joint space may remove these inflammatory mediators as well as alter the intra articular pressure through the addition of more fluid. Before arthrocentesis is performed, patients must undergo appropriate diagnosis and treatment planning. This is based upon history, visual analogue pain scales, and clinical and radiographic findings. Clinical examination should include inter incisal opening, lateral excursions, deviation on opening, and documentation of any occlusal discrepancies. Auscultation of the joint was performed to ascertain any click or crepitus, which may indicate disc displacement or degenerative joint disease, respectively. Radiographic imaging would initially include a panoramic screening film followed by other appropriately indicated methods..Patients were advised conservative therapy and those who failed to respond after two months ware candidates for arthrocentesis in this study.. Dynamic ultrasonography was done to assess the status of discs.

Past interest has been directed at the changes observed in the shape and position of the articular disc. More recent research has focused on the biomechanical and biochemical processes involved in temporomandibular joint dysfunction. Within the temporomandibular joint, surface active phospholipids function as lubricants and protectors of the articular surfaces. Lysis of these surface active phospholipids by phospholipase A2 can result in a significant increase in friction and adhesion between articular surfaces.

The etiology of pain associated with the temporomandibular joint is multifaceted. Possible sources of pain include impingement, compression, and inflammatory changes in the retrodiscal tissues; inflammatory changes in the synovial membrane with joint effusion; and capsulitis. Temporomandibular joint internal derangements have been described to progress through clinical stages, initially present as clicking with normal interincisal opening and progressing to limited opening, lacking translator movement where clicking ceases because of a non reducible disc.

Takafumi Hayashi's ${ }^{3}$ study on sonography revealed a sensitivity of $83 \%$, a specificity of $96 \%$, and an accuracy of $92 \%$ for identifying disk displacement. Rüdiger Emshoff etal ${ }^{4}$ study reveals that, when real-time images are interpreted by expert radiologists, dynamic sonography performed during maximal mandibular range of motion may provide valuable information about disk displacement of the TMJ.

Arthrocentesis of the temporomandibular joint was performed with approximately 100 milliliters of Ringers lactate solution. After completion of the lavage, the joint is manipulated by moving the patient's mouth through opening, protrusive, and excursive movements to free up the disc. According to literature a goal of 30 to 40 millimeters of inter incisal opening should be achieved at the completion of arthrocentesis. All the patients in both the study groups groups achieved more than 30 millimeters of inter incisal opening post operatively. Dynamic ultrasonography was performed by single observer, to assess the status of disc. Depending on the ultrasonographic finding, patients were categorized into two groups

Group 1- Patients without temporomandibular joint disc reduction Group 2- Patients with temporomandibular joint disc reduction

10 patients were included in each group. Analysis was done.

Age

Salvatore Sembronio ${ }^{2}$ reported an age range of 21 to 73 with a mean age of 41.8 years. G. Dimitroulis ${ }^{5}$ study group included an age range of 25 to 39 with an average of 32.5 years. In our study, the mean age of patients in group 1 is 38.6 \pm 11.3 and that of group 2 is $36.6 \pm 9$. Difference 
in mean age between the two groups is found to be statistically insignificant.

\section{Gender}

Temporomandibular joint internal derangements are more common in females. Current literature supports this gender difference. $90 \%$ of the patients in both groups were females. In our study, $90 \%$ of the patients in both group 1 and 2 were females.

\section{Visual analogue scale pain level}

Compared to group 2, there is a greater reduction of immediate post-operative pain, measured by VAS score, in group 1. Comparison of immediate post-operative pain, assessed by VAS score was statistically significant. In group 1 , there is a further reduction of pain on 1 week, 1 month and 6 month follow up. Three patients ( 1 male and 2 female) from group 1 experienced total relief of pain post operatively. In group 2, there was no significant reduction in the VAS score on postoperative follow up.

\section{Mouth opening}

Comparison of immediate post-operative inter incisal distance between the two groups was statistically insignificant. There was greater improvement in mouth opening in group 1 compared to group 2. In group 1 patients, there was improvement in mouth opening during postoperative follow up period.

\section{Lateral excursion}

Post-operative lateral excursion is more in group 1 than group 2 and the difference is statistically significant

\section{Conclusion}

This study was done to compare the outcome of arthrocentesis in patients with internal derangement of Temporomandibular joint with disc reduction and without disc reduction. Dynamic ultrasonography was found to be a useful imaging method for the diagnosis of disc displacement in patients with internal derangement of temporomandibular joint. It is noninvasive and less expensive to perform than any other imaging techniques. It can also be used in patients with claustrophobia and artificial pace makers. Patients can also avoid radiation hazards. In this study, 90 percent of the patients were females. A volume of 100 milliliters of Ringers lactate was sufficient to obtain a good lavage of the joint as shown by the increased mobility and decreased pain of the temporomandibular joint. The results showed a statistically significant difference in the outcome of arthrocentesis between the two groups and the patients without disc reduction responded to the procedure well. Post-operative dynamic ultrasonography revealed no change in the status of the disc in patients without disc reduction. So it may be concluded that even though there is a significant reduction in the symptoms associated with temporomandibular joint, arthrocentesis failed to release the closed lock of the articular disc. So, disc recapturing is not essential to obtain good function and relief of pain in patients with closed lock

\section{References}

1. Dorrit W. Nitzan D. Arthrocentesis Indication for Using This Minimally Invasive Approach for Temporomandibular Disorders. Oral Maxillofacial Surg Clin N Am. 2006;18:311-28.

2. Salvatore Sembronio M, a Alberto Maria Albiero, MD,bCorrado Toro, MD,c, Massimo Robiony M, FEBOMFS,d and Massimo Politi, MD, DMD,e Udine, Italy. Is there a role for arthrocentesis in recapturing the displaced disc in patients with closed lock of the temporomandibular joint? Oral surgery, Oral medicine, Oral pathology, Oral radiology, and Endodontology 2008. 2008; Vol. 105(No. 3):274-80.

3. Takafumi Hayashi JI, Jun-ichi Koyama, and Kazuhiro Yamada. The Accuracy of Sonography for Evaluation of Internal Derangement of the Temporomandibular Joint in Asymptomatic Elementary School Children: Comparison with MR and CT. 
AJNR Am J Neuroradiol 22.2001;22:72834

4. Emshoff R, Jank S, Bertram S, Rudisch A, Bodner G. Disk Displacement of the Temporomandibular Joint: Sonography Versus MR Imaging. AJR 2002.;178:1557

5. G. Dimitroulis MFD, A. Martinez. Temporomandibular joint arthrocentesis and lavage for the treatment of closed

6. Lukas M. Vos JJRHS, Boudewijn Stegenga,. Lavage therapy versus nonsurgical therapy for the Treatment of Arthralgia of the Temporomandibular Joint: A Systematic Review of Randomized Controlled Trials. J Orofac Pain 2013.2013;27:171-9

7. MarcelloMelis D, RPharm, Simona Secci MD, Caroline Ceneviz, DDS, MS. Use of ultrasonography for the diagnosis of temporomandibular joint disorders: A review. American Journal of Dentistry.2007;20(2):73-8.

8. LJ Pereira1 MGo,, LR Bonjardim and PM Castelo. Ultrasound and tomographic evaluation of temporomandibular joints in adolescents with and without signs and symptoms of temporomandibular disorders: a pilot study. Dento maxillofacial Radiology. 2007;36:402-8

9. Keiseki Kaneyama D, PhD, Natsuki Segami, DDS, PhD, Jun Sato D, PhD, Kazuma Fujimura, DDS, PhD, Toshikazu Nagao D, PhD, and, Hiroshi Yoshimura D, PhD. Prognostic Factors in Arthrocentesis of the Temporomandibular Joint: Comparison of Bradykinin, Leukotriene B4, Prostaglandin E2, and Substance P level in synovial fluid between successful and unsuccessful cases. Journal of oral and maxillofacial surgery: official journal of the American Association of Oral and Maxillofacial Surgeons. 2007;65:242-

10. Rüdiger Emshoff M, DMD,* and Ansgar Rudisch, MD $\dagger$. Temporomandibular joint internal derangement and osteoarthrosis:
Are effusion and bone marrow edema prognostic indicators for arthrocentesis and hydraulic distention. Journal of oral and maxillofacial surgery: official journal of the American Association of Oral and Maxillofacial Surgeons. 2007;65:66-73.

11. Demirhan Diraçog lu M, a I'lknur Bayraktar Saral, MD,aBarıs, Keklik, MD,b Hanefi Kurt, DT,c, UfukEmekli M, b LeventÖzçakar, MD,d,eAys, e Karan, MD,a and Cihan Aksoy, MD. Arthrocentesis versus nonsurgical methods in the treatment of temporomandibular disc displacement without reduction.Oral Surg Oral Med Oral Pathol Oral Radiol Endod. 2009;108:3-8.

12. Ezher Hamza Dayisoylua EC, Sina Uckan. Ultrasound-guided arthrocentesis of the temporomandibular joint. British Journal of Oral and Maxillofacial Surgery.2013; 51:667-8. 